\title{
THE INFLUENCE OF COMPONENTS CONCENTRATION IN THE ELECTRODEPOSITION PROCESS ON THE PROTECTIVE PROPERTIES OF 3,4-ETHYLENEDIOXYTHIOPHENE (EDOT) WITH 4-(PYRROLE-1-YL) BENZOIC ACID (PYBA), POLYOXYETHYLENE-10- LAURYLETHER (BRIJ) AND LITHIUM PERCHLORATE
}

\author{
Lidia ADAMCZYK¹, Agata DUDEK² \\ 1,2 Czestochowa University of Technology, Institute for Material Engineering, Faculty of Processing \\ Engineering and Applied Physics, Czestochowa, Poland, EU \\ adamczyk@wip.pcz.pl, dudek@wip.pcz.pl
}

https://doi.org/10.37904/metal.2019.881

\begin{abstract}
The electropolymerization of EDOT/PyBA/BRIJ by cyclic voltammetry on stainless steel and glassy carbon was studied in aqueous solution. The protective properties of the resulting coatings on stainless steel were investigated in the aggressive medium of $0.25 \mathrm{~mol} \mathrm{dm}^{-3} \mathrm{~K}_{2} \mathrm{SO}_{4}+0.5 \mathrm{~mol} \mathrm{dm}^{-3} \mathrm{KCl}$ with $\mathrm{pH}=2$. Diagnostic experiments included measurements of potentiodynamic polarization.
\end{abstract}

Keywords: Poly(3,4-ethylenedioxythiophene), 4-(pyrrole-1-yl) benzoic acid, polyoxyethylene-10-laurylether, composite coatings, electrodeposition, corrosion, chloride media

\section{INTRODUCTION}

The use of conducting polymers (CP) is systematically growing with respect to protecting metals against corrosion [1,2] and also for more advanced applications such as in microelectronics and chemical sensing [1]. In such applications, CP are used as coatings for metals or serve as a substrate to metallic layers. In both cases, metal/polymer interface affects the global material properties. During the last few years, effort has been devoted to the electrodeposition of CP on oxidisable metals and good results have been obtained mainly in organic solvents. Two types of approaches are currently followed. The first one concerns the modification of the polymerization conditions such as solvents, $\mathrm{pH}$, and monomer concentration [3-6] while the second one proceeds with chemical modification of the surface, for instance by coupling agents, before electropolymerization process [7-10]. Combining the two approaches could also be an attractive way to investigate. Following these first approach, Sakmeche et al. [11], proposed recently the use of an aqueous anionic micellar medium containing sodium dodecylsulfate (SDDS) for electropolymerization. Under such conditions they showed a decrease of 3,4-ethylenedioxythiophene (EDOT) oxidation potential on platinum compared with the approach using organic solvents. The lower the monomer oxidation potential, the better it is for the polymerization especially when the substrate is an oxidisable metal (e.g. zinc, iron, titanium, aluminium). An important limitation on the use of PEDOT coatings is their unsatisfactory adhesion to the metal (steel) substrate and, consequently, limited protection capability. This arouses the need for seeking methods for improving the adhesion of the polymer to the substrate. Another obstacle to the common use of EDOT is a poor solubility of its monomer in water (a mere $2.1 \mathrm{~g} \mathrm{dm}^{-3} 20^{\circ} \mathrm{C}$ ), which restricts the effectiveness of the polymerization process and increases the coating build-up time. The introduction of surface-active compounds to the modifying solution was aimed at increasing the degree of dispersion of the monomer (EDOT) in this solution, which favourably influenced the deposition process effectiveness. To increase the monomer concentration in the water solution, polyoxyethylene glycol monolaurate ether (BRJI) was used as a surfactant. A surfactant-containing solution is micellar in character with a considerably increased effective EDOT content lowers the monomer oxidation potential and has a favourable effect on the number of active polymerization sites $[12,13]$. $\mathrm{LiClO}_{4}$ was additionally introduced to the modifying solution. Perchlorate ions are inhibitors of the corrosion of iron and its alloys. 
It has been observed in earlier studies [14-16] that a compound that exhibits good adhesion to the steel substrate and, at the same time, has not been used so far in PEDOT compounds is benzopyrrolic acid. Coatings obtained using this compound are characterized by strong adhesion to the substrate and a uniform, stable form. In addition, due to the presence of the readily dissociating carboxyl group, the PyBA coatings undergo modification by attaching the positive ions from the oxidized PEDOT. The study has investigated the effect of PEDOT, BRIJ concentration in the modifying solution and the influence of PEDOT/PyBA/BRIJ coatings depositing time and potential polarization rate on the protective properties of the obtained coatings. The protective properties of PEDOT/PyBA/BRIJ were examined in solutions containing sulphate and chloride ions, acidified to $\mathrm{pH}=2$. Chloride ions were selected in order to determine their effect on the local corrosion of passivating metallic materials.

\section{MATERIALS AND METHODS}

All chemicals were of analytical grade purity. The electropolymerization of the best coating PEDOT/PyBA/BRIJ was performed in an aqueous microemulsion consisting of $0.1 \mathrm{~mol} \mathrm{dm}^{-3}$ 3,4-ethylenedioxythiophene/EDOT (Aldrich), $10 \mathrm{mg}$ of 4-pyrrole-1-yl) benzoic acid/PyBA in $1 \mathrm{dm}^{-3}$ water (Aldrich), $0.04 \mathrm{~mol} \mathrm{dm}^{-3}$ polyoxyethylene10-laurylether/BRIJ surfactant (Aldrich) and $0.1 \mathrm{~mol} \mathrm{dm}^{-3} \mathrm{LiClO}_{4}$ (Aldrich). In order to select optimal conditions for coating deposition, the electropolymerization process was also carried out with solutions: a) $0.04 \mathrm{~mol} \mathrm{dm}^{-3}$ BRIJ + $0.1 \mathrm{~mol} \mathrm{dm}^{-3} \mathrm{LiClO}_{4}+10 \mathrm{mg} \mathrm{w} 1 \mathrm{dm}^{3}$ PyBA + EDOT (with concentrations: 2, 10, 30, 68, $100 \mathrm{mmol} \mathrm{dm}^{-}$ 3); b) $10 \mathrm{mg} \mathrm{w} 1 \mathrm{dm}^{3}$ PyBA $+0.1 \mathrm{~mol} \mathrm{dm}^{-3} \mathrm{LiClO}_{4}+0.1 \mathrm{~mol} \mathrm{dm}^{-3}$ EDOT + BRIJ (with concentrations: 5, 10, 40, $100 \mathrm{mmol} \mathrm{dm}^{-3}$ ). They were recrystallized before use. Deionized water was used as a solvent to prepare solutions for modification and electrolytes. Experiments were carried out at room temperature $\left(20+2{ }^{\circ} \mathrm{C}\right)$. Due to the low solubility of EDOT and PyBA monomers in water, surfactant BRIJ was used to increase the concentration of both components in the medium. The use of monomer alone will limit the effectiveness of the polymerization process and extend the coating build-up time. The surfactant introduced to the solution lowers the monomer oxidation potential [16]. The composite coatings of PEDOT/BRIJ/PyBA with $\mathrm{LiClO}_{4}$ and were grown on glassy carbon and stainless steel electrode substrates by potential cycling (at $50 \mathrm{mVs}^{-1}$ ). Typically, 16 full potential cycles, starting from -0.40 and ending at $1.20 \mathrm{~V}$, were applied.

Electroctrochemical measurements were performed using $\mathrm{CH}$ Instruments (model $\mathrm{CHI} 660$ ) workstation (Austin, USA). A glassy carbon disk (geometric area, $0.2 \mathrm{~cm}^{2}$ ) from Mineral (Warsaw, Poland) or X20Cr13 stainless steel (C-0.17\%; Cr-12.6\%; Si-0.34\%; Ni-0.25\%; Mn-0.30\%; V-0.04\%; P-0.024\%, S<0.005\%) disk (geometric area, $0.2 \mathrm{~cm}^{2}$, embedded in epoxy resin) acted as a working electrode. Before modification, a glassy carbon electrode was activated by polishing with fine grade aqueous alumina slurries (grain size, $5 \pm 0.5$ $\mu \mathrm{m}$ ) on a polishing cloth. Experiments were done in conventional three- electrode system, where a platinum wire served as a counter electrode and a saturated $(\mathrm{KCl}) \mathrm{Ag} / \mathrm{AgCl}$ electrode as a reference electrode. Potentiodynamic logarithm (current density) - potential curves were recorded at a fairly low, $10 \mathrm{mV} \mathrm{s}^{-1} \mathrm{scan}$ rate, in $0.25 \mathrm{~mol} \mathrm{dm}^{-3} \mathrm{~K}_{2} \mathrm{SO} 4+0.5 \mathrm{~mol} \mathrm{dm}^{-3} \mathrm{KCl}$ with $\mathrm{pH}=2$ and $0.25 \mathrm{~mol} \mathrm{dm}^{-3} \mathrm{~K}_{2} \mathrm{SO}_{4}$ with $\mathrm{pH}=2$ electrolyte.

\section{RESULTS AND DISCUSSION}

PEDOT/PyBA/BRIJ coatings were deposited for a duration of $1000 \mathrm{~s}$, making 16 deposition cycles during that period of time. Figures $1 \mathrm{~A}$ and $1 \mathrm{~B}$ show voltammetric curves for depositing a coating on glassy carbon and stainless steel $\mathrm{X} 20 \mathrm{Cr} 13$ from the PEDOT/PyBA/BRIJ solution. In the first cycles, a gradual coating build-up is observed, which manifests itself in an increase in oxidation and reduction currents. In the next potential sweep cycles, the coating build-up is much slower, the shape of the peaks changes, and the current density magnitudes decrease. It is hard to unambiguously explain the observed decline in electroactivity. It is likely that for thicker coatings the electropolymerization leads to stable polymeric structures with PEDOT, while the decrease in electroactivity for longer deposition duration might be due to a limited stability of external PEDOT structures (incomplete polymerization). In the first cycle of depositing the PEDOT/PyBA/BRIJ composite 
coating of steel X20Cr13 (Figure 1B), the oxidation of the steel surface takes place. The steel becomes passivated from the moment of starting the measurement, that is from the potential $-0.2 \mathrm{~V}$. The increase in current density above the potential $0.8 \mathrm{~V}$ may indicate both the start of the polymer oxidation process, as well as the process of steel transpassivation (involving the chromium oxidation $\mathrm{Cr}(\mathrm{III}) \rightarrow \mathrm{Cr}(\mathrm{IV})$ ). The microstructures of the polymer and benzopyrrolic acid interact electrostatically between one another, because the oxidized PEDOT is charged positively and PyBA has a readily dissociating carboxyl group. The latter characteristic leads to an enhanced stability of the composite coating. Adding the surfactant to the modifying solution reduced the monomer oxidation potential and had a favourable effect on the number of active polymerization sites [16].
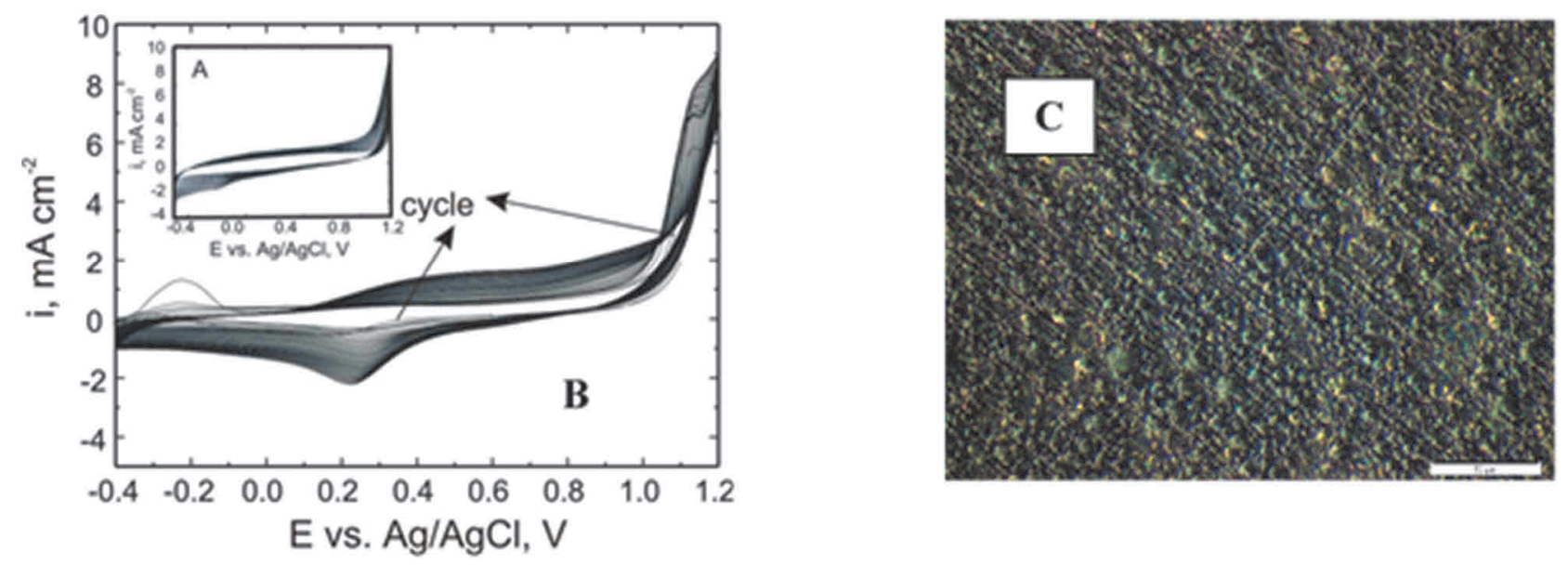

Figure 1 Voltammetric generation illustrating electrodeposition of PEDOT/PyBA/BRIJ on glass carbon (A), PEDOT/PyBA/BRIJ coating on stainless steel (B) and SEM image of PEDOT/PyBA/BRIJ coating on stainless steel (C) from the solutions for modification. Scan rate $50 \mathrm{mV} \mathrm{s}^{-1}$. Electrode geometric area $0.2 \mathrm{~cm}^{2}$.

Immediately after depositing polymeric coatings on a glassy carbon electrode (Figure 1A) from modifying solutions with varying PEDOT concentrations, cyclic voltammetric curves were recorded in a $0.5 \mathrm{~mol} \mathrm{dm}^{-3}$ $\mathrm{H}_{2} \mathrm{SO}_{4}$ solution using a polarization rate of $50 \mathrm{mV} \mathrm{s}^{-1}$ (Figure 2). In voltammetric response measurements, the course of the first cycle was always represented. Figure 2 shows curves recorded in the potential interval from $-0.2 \mathrm{~V}$ to $1.2 \mathrm{~V}$. No significant changes in recorded current magnitudes during potential sweeping was noted in this interval. Neither anodic nor cathodic peaks (i.e. oxidation and reduction peaks) for PEDOT/PyBA/BRJI were observed. Such a behaviour suggests a capacitive characteristic of the coatings. Literature reports describing PEDOT show that in non-aqueous solutions oxidation and reduction peaks lie within the potential interval from $-0.2 \mathrm{~V}$ to $0.9 \mathrm{~V}$ [9], while for PyBA no characteristic peaks have been found. The voltammetric curves in Figure 2 show a very good symmetry, the charges of the cathodic part are equal to the charges of the anodic part, which indicates a reversibility of the oxidation and reduction reactions in the potential interval from -0.2 to $1.2 \mathrm{~V}$. The highest current densities in the voltammetric responses can be observed for the coating

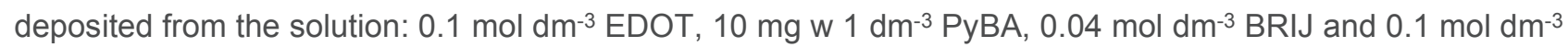
$\mathrm{H}_{2} \mathrm{SO}_{4}$; this is indicative of an increase in coating mass.

Figure 3 illustrates voltammetry responses recorded on glassy carbon after the immediate deposition of coatings from a solution of varying BRIJ concentration. Metallographic observations and the examination of coating adhesion to the substrate have shown that the best properties are exhibited by a coating with a BRIJ concentration of $0.4 \mathrm{~mol} \mathrm{dm}^{-3}$ in the modifying solution. At a higher BRIJ content in the modifying solution, dense colloid forms, from which it is very difficult to deposit a coating, while at lower BRIJ concentrations no decrease in EDOT monomer oxidation potential is observed. 


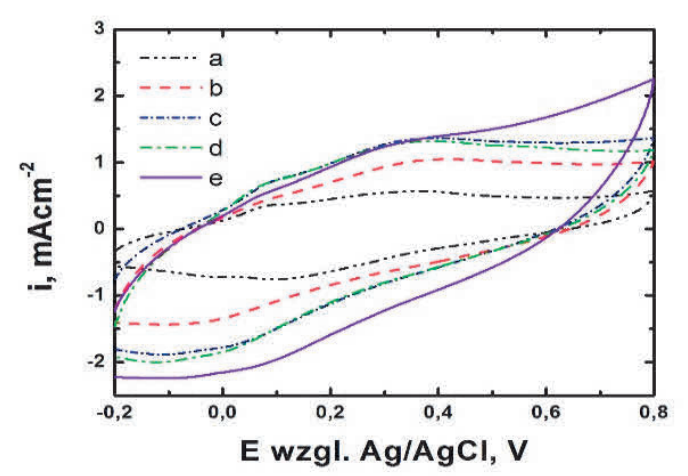

Figure 2 Response voltammetry of the PEDOT/PyBA/BRIJ coatings on the glassy carbon in solutions: $10 \mathrm{mg} \mathrm{w}^{1} \mathrm{dm}^{3}$ PyBA+ $0.04 \mathrm{~mol} \mathrm{dm}^{-3}$ BRIJ + $0.1 \mathrm{~mol} \mathrm{dm}^{-3} \mathrm{LiClO}_{4}$ and (a) 2, (b) 10, (c) 30, (d) 68 and (e) $100 \mathrm{mmol} \mathrm{dm}^{-3}$ EDOT. Elecrolyte: $0.5 \mathrm{~mol} \mathrm{dm}^{-3} \mathrm{H}_{2} \mathrm{SO}_{4}$. Scan rate: $50 \mathrm{mV} \mathrm{s}^{-1}$.

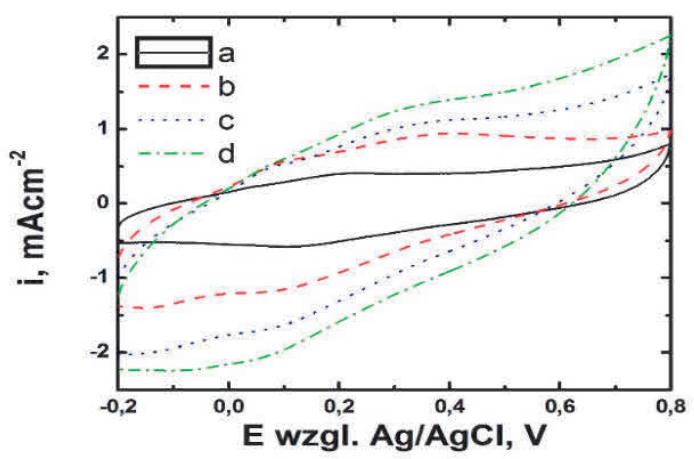

Figure 3 Response voltammetry of the PEDOT/PyBA/BRIJ coatings on the glassy carbon

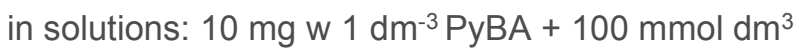
EDOT + $0.1 \mathrm{~mol} \mathrm{dm}^{-3} \mathrm{LiClO}_{4}$ and (a) 5, (b) 10, (c) 40 and (d) $100 \mathrm{mmol} \mathrm{dm}^{-3}$ BRIJ. Elecrolyte: $0.5 \mathrm{~mol}$ $\mathrm{dm}^{-3} \mathrm{H}_{2} \mathrm{SO}_{4}$. Scan rate: $50 \mathrm{mV} \mathrm{s}^{-1}$.

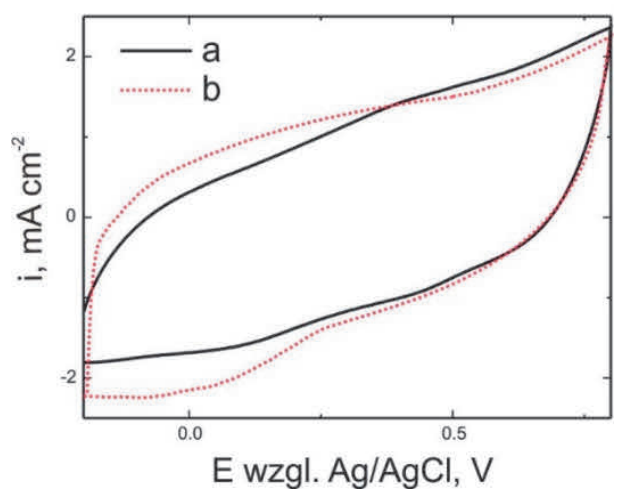

Figure 4 Response voltammetry of the PEDOT/PyBA/BRIJ coatings on the glassy carbon (a) and stainless steel (b). Electrolyte: $0.5 \mathrm{~mol} \mathrm{dm}^{-3} \mathrm{H}_{2} \mathrm{SO}_{4}$. Scan rate: $50 \mathrm{mV} \mathrm{s}^{-1}$.

Figure 4 shows cyclic voltammetry curves recorded in the potential interval from $-0.2 \mathrm{~V}$ to $0.8 \mathrm{~V}$. No significant changes in recorded current magnitude was noted in this interval during potential sweeping. No clear anodic or cathodic peaks (i.e. oxidation and reduction peaks) for PEDOT and PyBA were observed. As can be noticed, the anode and cathode current densities depend on the electrode substrate. The voltammetric curves recorded for coatings produced on glassy carbon show smaller cathode and anode current densities, compared to the curves recorded for coatings deposited on steel X20Cr13.

The corrosion potentials for steel X20Cr13 coated with a PEDOT/PyBA/BRIJ are shifted by approx. $0.2 \mathrm{~V}$ towards positive values (Figures 5,6 ), as against the corrosion potential value recorded for the uncoated steel $\left(E_{c o r}=-0.63 \mathrm{~V}\right)$. For the steel protected with a coating (Figure 3 ), the current densities in the passive range are slightly lower than those for the uncoated steel. In the presence of a composite coating, a lowering in transpassive dissolution current density is observed $(E>0.09 \mathrm{~V})$. Above $E \approx 0.0 \mathrm{~V}$, however, pitting of the passive coating is observed for both the uncoated and coated steel, although the $E_{\text {pit }}$ value is slightly more positive in the presence of a coating (Figure 6). Moreover, in the presence of the PEDOT/PyBA/BRIJ coating, the pitting development is much less intensive, as evidenced by a clearly smaller steepness of the curve above $E_{\text {pit }}$ for a specimen coated with the coating. In the presence of chloride ions, an inhibition of active dissolution 
by the PEDOT/PyBA/BRIJ coating is visible. The above-mentioned observations allow one to believe that composite coatings containing an organic polymer and benzopyrrolic acid of ion-exchange properties make the access by chloride ions to the substrate difficult and improve the effectiveness of protection against atmospheric and local corrosion. Introducing an anion with a negative charge to the positively charged matrix of a conducting polymer results in a neutralization of the anion-exchange (membrane) properties of the coating. An added advantage of such coatings is their very good adhesion to the coating substrate.

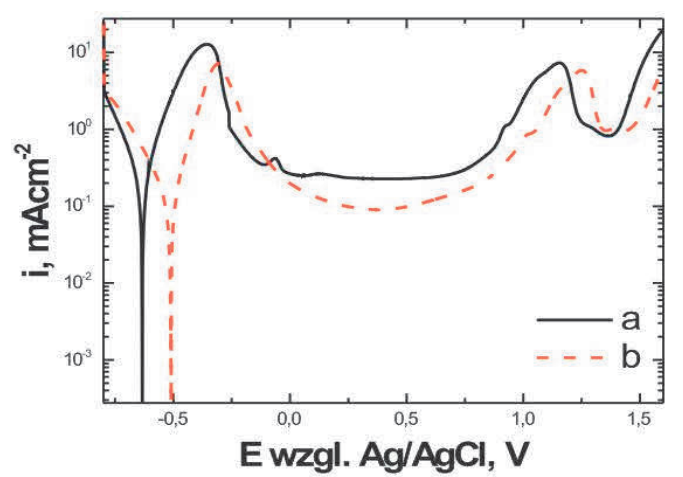

Figure 5 Potentiodynamic polarization curves recorded in an acidified $(\mathrm{pH}=2)$ solution of $0.25 \mathrm{~mol}$ dm-3 K2SO4 for uncoated X20Cr13 stainless steel (a), and coated with composite PEDOT/PyBA /BRIJ (b). Scan rate $10 \mathrm{mV} \mathrm{s}-1$.

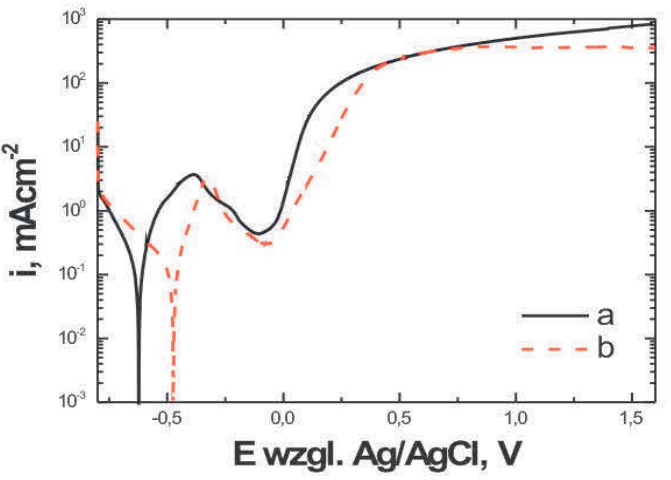

Figure 6 Potentiodynamic polarization curves recorded in an acidified $(\mathrm{pH}=2)$ solution of $0.25 \mathrm{~mol}$ dm-3 K2SO4 + $0.5 \mathrm{~mol} \mathrm{dm}-3 \mathrm{KCl}$ for uncoated

$\mathrm{X} 20 \mathrm{Cr} 13$ stainless steel (a), and coated with composite PEDOT/PyBA/BRIJ coatings. Scan rate $10 \mathrm{mV} \mathrm{s}-1$.

\section{CONCLUSIONS}

We have demonstrated the possibility of modification of a stainless steel substrate with a PEDOT/PyBA/BRIJ based coatings. Such coatings exhibit promising properties toward the protection of stainless steel against general and pitting corrosion in the chloride-containing medium. The results are consistent with the view that the PEDOT/PyBA/BRIJ composite coatings is not only characterized by good physicochemical stability (understood in terms of resistance to degradation during repetitive potential cycling) and compact (dense) morphology but it is also capable of effective propagation of charge (that could be useful in mediation of various redox reactions). Regardless the nature of interactions between PEDOT and PyBA components, the composite PEDOT/PyBA/BRIJ system cannot be considered as a simple mixture of these components. Our results indicate the usefulness of PEDOT/PyBA/BRIJ coatings for the protection against general and pitting corrosion of stainless steels in moderately acidic $(\mathrm{pH}=2)$ sulfate media.

\section{REFERENCES}

[1] TALLMAN Dennis E., SPINKS, Geoff, DOMINIS, Anton, WALLACE, Gordon G. Electroactive conducting polymers for corrosion control, J Solid State Electrochem, 2002. vol. 6, pp. 73-84,

[2] DESHPANDE, Pravin P., SAZOU, Dimitra, Corrosion Protection of Metals by Intrinsically, Conducting Polymers", CRC Press, Taylor \& Francis Group. 2016,

[3] HEINZE, Jürgen, FRONTANA-URIBE, Bernardo A., LUDWIGS, Sabine, Electrochemistry of Conducting Polymers-Persistent Models and New Concepts. Chemical Reviews. 2010, 110 (8), pp 4724-4771

[4] WALLACE, Gordon.G., TEASDALE, Peter R., SPINKS, Geoff, KANE-MAGUIRE, Lab. Conductive Electroactive Polymers: Intelligent Materials Systems, Second Edition, CRC Press, Taylor \& Francis Group M. Schirmeisen. 2003. 
[5] PRATHISH, Krishna P., CARVALHO, Ricardo C., BRETT, Christopher M.A. Highly sensitive poly(3,4ethylenedioxythiophene) modified electrodes by electropolymerisation in deep eutectic solvents. Electrochemistry Communications. 2014. vol. 44, pp. 8-11

[6] GROENENDAAL, L. 'Bert', ZOTTI, G., AUBERT, P.-H., WAYBRIGHT., S.M. Electrochemistry of poly(3,4alkylenedioxythiophene) derivatives. Advanced Materials. 2003. vol. 15, Issue 11, pp. 855-879

[7] BUREAU, Christophe, DELHALLE Joseph. Synthesis and structure of polymer/metal interfaces: a convergence of views between theory and experimental. Journal of surface Analysis. 1999. vol. 6. no.2, pp.159-170

[8] MEKHALIF, Zineb, DELHALLE, Joseph, LANG, Philippe, GARNIER, Françis, PIREAUX, Jean-Jacques. Comparative study of the electrodeposition of polybithiophene films on titanium electrodes: bare and modified with aromatic and aliphatic thiols. Synthetic Metals. 1998. vol. 96, pp.1165-175.

[9] LANG, Philippe, MEKHALIF, Zineb, GARNIER, Francis. Blocking and chemical pretreatments of platinum electrode for the electropolymerization of thiophene. J. Chim. Phys. Physicochem.Biol. Spec. Issure. 1992. vol.89 1063-1070.

[10] MEKHALIF, Zineb, LANG, Philippe, GARNIER, Francis. Chemical pretreatment of platinum by aromatic and aliphatic thiols. Effect on polybithiophene electrodeposition and properties. Journal of Electroanalytical Chemistry. 1995.vol.399, pp. 61-70.

[11] SAKMECHE Nacer, AARON, Jean-Jacques, AEIYACH Salah, LACAZE, Pierre Camille. Usefulness of aqueous anionic micellar media for electrodeposition of poly-(3,4-ethylenedioxythiophene) films on iron, mild steel and aluminium. Electrochimica Acta. 2000. vol.45, no.12, pp.1921-1931

[12] BHANDARI, Shweta, DEEPA, Melepurath SINGH, Sukhvir C., GUPTA, Govind, KANT, Rama, Redox behavior and optical response of nanostructured poly(3,4-ethylenedioxythiophene) films grown in a camphorsulfonic acid based micellar solution. Electrochimca Acta. 2008. vol. 53, no.7, pp. 3189-3199.

[13] KIM, Taeyoung, KIM, Jongeun, KIM, Yunsang, LEE, Taehee, KIM, Wonjeong, SUH, Kwangsuck. Preparation and characterization of poly(3,4-ethylenedioxythiophene) (PEDOT) using partially sulfonated poly(styrene-butadienestyrene) triblock copolymer as a polyelectrolyte. Current Applied Physics. 2009. vol. 9, no.1, pp. 120-125.

[14] ADAMCZYK, Lidia, GIZA, Krystyna, DUDEK, Agata, Electrochemical preparation of composite coatings of 3,4etylenodioxythiophene (EDOT) and 4-(pyrrole-1-yl) benzoic acid (PyBA) with heteropolyanions. Materials Chemistry and Physics. 2014. vol. 144, no. 3, pp. 418-424

[15] ADAMCZYK, Lidia, Aplication of composite films coating poly(3,4-ethylenodioxytiophene and silicotungistic acid with 4-(pyrrole-1-yl) benzoic acid for protection of X20Cr13 steel against corrosion. Ochrona przed Korozją. 2010. vol.53, no. 4-5, 282-285

[16] Adamczyk, Lidia, Kulesza, Paweł J., Fabrication of Composite Coatings of 4-(Pyrrole-1-yl) Benzoate - Modified Poly-3,4-Ethylenedioxythiophene with Phosphomolybdate and Their Application in Corrosion Protection, Electrochimica Acta. 2011. vol. 56, no. 10, pp. 3649-3655. 